\title{
The effects of personal background and occupational stress on the QOL of Vietnamese care attendants working at medical institutions in Taiwan
}

\author{
Hsiu-Chen Chang Chien ${ }^{1}$, Su-Feng Chu ${ }^{2}$, Chi Chang ${ }^{2}$, Chien-An Sun ${ }^{3}$, Yu-Ching Chou ${ }^{4}$, \\ Shu-Chun Hsueh ${ }^{5}$, Tsan Yang ${ }^{5,}$, Tsan Yang ${ }^{6}$ \\ ${ }^{1}$ Respiratory Care Ward, Chien-Yu Hospital, Kaohsiung, Taiwan \\ ${ }^{2}$ Department of Nursing, Meiho University, Pingtung, Taiwan \\ ${ }^{3}$ Department of Public Health, College of Medicine, Fu-Jen Catholic University, New Taipei City, Taiwan \\ ${ }^{4}$ School of Public Health, National Defense Medical Center, Taipei City, Taiwan \\ ${ }^{5}$ Department of Health Business Administration, Meiho University, Pingtung, Taiwan \\ ${ }^{6}$ Department of Health Business Administration, Meiho University, Ping Kuang Road, Neipu, Pingtung, 91202, Taiwan, ROC
}

Email address:

tsan.yang@msa.hinet.net(T. Yang)

To cite this article:

Hsiu-Chen Chang Chien, Su-Feng Chu, Chi Chang, Chien-An Sun, Yu-Ching Chou, Shu-Chun Hsueh, Tsan Yang, Tsan Yang. The Effects of Personal Background and Occupational Stress on the QOL of Vietnamese Care Attendants Working at Medical Institutions in Taiwan. American Journal of Nursing Science. Vol. 2, No. 4, 2013, pp. 40-49. doi: 10.11648/j.ajns.20130204.11

\begin{abstract}
Background: With the rapid increase in the elderly population, there is a growing demand for care attendants at medical institutions in Taiwan The rapid growth in the number of foreign care attendants is significant and the quality of care provided by these foreign care attendants is directly related to the quality of care received by elderly people. However, few studies have addressed the relationship between occupational stress and quality of life (QOL) for foreign care attendants in Taiwan. Purpose: This study explored the relations between personal background and occupational stress and QOL of Vietnamese care attendants in Taiwan. Methods: This cross-sectional study recruited Vietnamese care attendants currently working at regional hospitals in Kaohsiung City and County who had worked in Taiwan for at least three months. We distributed a structured questionnaire to potential participants and collected 264 valid completed questionnaires between January and April 2010.Results: Significant statistical differences were detected in the QOL of Vietnamese care attendants of various ages and educational levels, in the number of non-work days in a month, the number of daily work hours, and the number of patients cared for. A negative correlation was found between the various levels of occupational stress and the eight dimensions of wellness for Vietnamese care attendants, indicating that higher occupational stress led to lower QOL. In addition, we developed a regression model for overall QOL (SF-36), a physical component summary (PCS) and a mental component summary (MCS). By entering the variables "workload and work procedures," "number of daily work hours," and "relationships with management supervisors" into our regression model, we calculated the variance for “overall QOL (SF-36)," "QOL for PCS," and "QOL for MCS" to be 44.9\%, 38.3\%, and 41.7\%, respectively. Conclusions: Higher levels of occupational stress led to a lower QOL for Vietnamese care attendants. Of all the factors, "workload and work procedures," "number of daily work hours," and "relationships with management supervisors" wielded the greatest influence on QOL.
\end{abstract}

Keywords: Medical Institution, Vietnamese Care Attendants, Occupational Stress, Quality of Life

\section{Introduction}

With the rapid increase in the elderly population in Taiwan, and the drastic changes in work environments and family values, families have found themselves no longer capable of providing long-term personal care for older family members, resulting in the eventual hiring of care attendants. This has led to a growing demand for care attendants at medical institutions. Although care attendants are among the most indispensable of all nursing staff, they have, in recent years, been replaced by foreign care attendants. The quality of care provided by these foreign 
care attendants is directly related to the quality of care received by elderly people $[1,2]$.

The growth in the number of Vietnamese care attendants is currently the most significant among all those of other foreign attendants, expanding from 2,634 in 2000 to 22,676 in 2009. Language barriers, differences in culture and custom, and limited professional expertise have contributed to the occupational stress perceived by Vietnamese care attendants inside medical institutions. Previous studies reported that $59.2 \%$ of these foreign care attendants possessed no prior nursing background, which has created problems inpatient-caregiver communication and in the assistants' ability to make decisions when working in Taiwanese hospitals. These problems have resulted in additional stress when providing nursing care [3]. As a consequence, not only does long-term occupational stress disrupt the physical and mental health of the caregivers, but it also disrupts their quality of life (QOL). If medical institutions understand the stress experienced by their staff and their basic needs and values, then these institutions would be able to provide necessary measures to improve workplace morale and instill a willingness to work, thereby enhancing the overall quality of care attendants $[4,5]$.

Since foreign care attendants were first allowed to work in Taiwan in 1992, the government has provided limited on-the-job training and support. The vast majority of these care attendants are asked to complete pre-job training in brokerage agencies prior to working in Taiwan. Pre-job training includes language and nursing care training [6], in which veteran foreign care attendants working for the agency are assigned to train new foreign care attendants on arriving in Taiwan. This results in many foreign care attendants partaking in heavy labor care work without professional pre-job or on-the-job training, or in some cases, without any job training at all. The combination of poor wages and benefits, the language barriers, and the need to provide nursing care without adequate skills easily creates work overload and negatively influences QOL [1, 6-9]. Labor health problems that stem from occupational stress have garnered wide attention in recent years. Not only does occupational stress disrupt mental health but it also disrupts physical health and QOL. Therefore, studies on the negative impact of occupational stress should focus not only on mental and physical health but also on the effects they have on QOL [10-13].

Recent studies on Vietnamese care attendants working in medical institutions have often explored the causes of occupational stress [8], job satisfaction [14,15], and methods for alleviating occupational stress and improving personal health [9]. Studies that have examined the relationship between occupational stress and QOL were only included health care workers, teachers, company executives, high-tech employees, and primary caregivers [11-13, 16, 17], however, Vietnamese care attendants were excluded. Therefore, this study was designed to examine the backgrounds and job responsibilities of these Vietnamese care attendants and explore the effects of occupational stress on their QOL.

\section{Methods}

\subsection{Study Design and Subject Selection}

This study employed a cross-sectional study design, sampling Vietnamese care attendants who had participated in actual care work for a minimum of three months in a district hospital in Kaohsiung City, and who possessed a basic command of the Chinese language. The list of workers was provided by a certified foreign workers' brokerage agency. The study received approval from the Meiho University Institutional Review Board before phone calls were made to the district hospital that consented to participate in this experiment. Interviewees were debriefed to explain the purpose and characteristics of the study. Questionnaires were completed after a consent form was signed by the participants. The research period lasted four months, from January to April of 2012.The questionnaires were collected by the researcher in this study with two staff members from the foreign workers' brokerage agency (one of whom was a translator). Together, they visited the medical institution on the date of the care attendants' salary payment to collect the completed questionnaires. For those who had difficulty answering the questions, simple explanations were provided by the accompanying translator A total of 300 questionnaires were distributed to female care attendants and 270 were returned. Excluding incomplete questionnaires, there were 264 actual valid questionnaires, resulting in a response rate of $88.0 \%$.

\subsection{Research Instruments}

Research data were gathered by administering the "Vietnamese Care Attendant Personal Background and Job Responsibilities Check Questionnaire, "the "Work Stressor Inventory," and the "SF-36 Quality of Life Scale." Among these data collection instruments, the new Work Stressor Inventory, based on the original Work Stressor Inventory scale developed by Lin (2000) [9] in accordance with the concept of Schaefer and Moos (1993) [18], consisted of 51 questions used to evaluate occupational stress in which the Cronbach's $\alpha$ was set at 0.80 , including pressure experienced to fulfill particular tasks: 18 questions addressed general job and nursing care responsibilities; 15 questions addressed stress from interpersonal relationships(e.g., among colleagues and management supervisors); 18 questions addressed systematic stress (i.e., workload and work procedures, as well as planning and sustaining the institutional environment). This inventory used a Likers five-point scale, in which higher scores indicated a higher level of stress. In addition, this study received consent and authorization to use the SF-36 Quality of Life Scale (Taiwanese-version), a self-administered questionnaire in which the Cronbach's $\alpha$ was set at 0.70 . The questionnaire asks the respondent to reflect on the previous four weeks and consider a total of eight 
dimensions, including "physical functioning" (PF; 10 questions), "role limitation because of physical problems" (RP; 4 questions), "bodily pain" (BP; 2 questions), "general health" (GH; 5 questions), "vitality" (VT; 4 questions), "social functioning" (SF; 2 questions), "role limitation caused by emotional problems" (RE; 3 questions), and "mental health" (MH; 5 questions). Subtotals were added in each of the eight dimensions before all were tallied. Some questions required the score to be inversed or weighted before being added to the total. The eight dimensions could be separated further to reflect two comprehensive scoring systems, namely the physical component summary (PCS) and the mental component summary (MCS).The score ranged from 0 to 100 , in which a score of 0 meant the poorest QOL, and a score of 100 meant the strongest QOL. In the end, a higher score revealed a higher QOL [19].

In consideration of the language differences, a bilingual expert specializing in translating Chinese to Vietnamese was hired to translate the structured questionnaire into Vietnamese prior to the experiment. After completion, a bilingual Vietnamese expert who majored in Chinese was hired to translate the structured questionnaire back to Chinese, allowing the researchers in this study to match and review the accuracy of its content. Then, Vietnamese care attendants who were able to read Chinese were asked to answer the questionnaire and provide insights into areas requiring further revision.

\subsection{Data Analysis}

The research data were processed and statistically analyzed using SPSS for Windows Version 17.0,in which the significance level was set at $\alpha=.05$. Frequency distribution, percentile, mean, and standard deviation were used to illustrate the distribution of Vietnamese care attendants based on their personal backgrounds and job responsibilities. In addition, the Student's t test and oneway ANOVA were used to evaluate the different personal backgrounds and job responsibilities of these Vietnamese care attendants, as well as the occupational stress they had experienced and their QOL. Further, stepwise multiple regression analysis was used to examine the factors that affected QOL.

\section{Results}

\subsection{Relationship between Personal Backgrounds and Occupational Stress of Vietnamese Care Attendants}

The 264 respondents who participated in this study were exclusively female. Table 1 showed that the younger group tended to experience more occupational stress than did the older group. Levels of stress encountered also differed significantly for care attendants of different educational levels. Using Scheffe's post hoc analysis, we observed that those who graduated from college experienced higher levels of occupational stress than did those who had completed only middle school, high school, or vocational school. Years of service also resulted in significant differences in "overall occupational stressor," and stress from "general job responsibilities," "workload and work procedures," and "planning and sustaining the institutional environment." Those with over 2 years of experience experienced more occupational stress than did those who had worked less than 2 years. Those who had performed similar nursing care duties in Taiwan experienced more occupational stress in "relationships with management supervisors" than did those who had not. In terms of the number of non-work days in a month, only "relationships with colleagues" showed non-significant difference; while significant differences were observed in all other categories. The post hoc analysis clearly showed how fewer vacation days in a month resulted in much higher levels of occupational stress. Regarding daily work hours, only "nursing care responsibilities" showed no significant difference; significant differences were observed in all other categories. Those working 11-12 hours per day also experienced more occupational stress than did those working 8-10 hours. "Overall occupational stressor" differed significantly for the different numbers of patients cared for during the day and night, showing that the care attendants with 16 patients and more experienced occupational stress than did those with 11-15 and those with 6-10. Those who had not taken nursing care training courses in Vietnam experienced higher levels of occupational stress in their "relationships with management supervisors" than did those who had received training in Vietnam. Those who had received on-the-job training in Taiwan experienced much greater occupational stress in "overall occupational stressor," "nursing care responsibilities," "relationships with colleagues," and "relationships with management supervisors. "Those who had taken shelter next to patients experienced higher levels of occupational stress in "nursing care responsibilities" than did those being assigned a room by the institution. Those whose living environments were rated as loud and unclean experienced greater occupational stress in their "relationships with management supervisors" than did those whose living environments were rated as neat and clean. 
Table 1. Personal backgrounds of Vietnamese care attendants and analysis of the differences among various dimensions of occupational stress $(N=264)$

\begin{tabular}{|c|c|c|c|c|c|c|c|c|c|c|c|c|c|c|c|}
\hline Variables & $\mathbf{N}$ & $\begin{array}{l}\text { overall } \\
\text { occupatio } \\
\text { nal } \\
\text { stressor } \\
\text { Mean } \pm \text { SD }\end{array}$ & $\begin{array}{l}t / F / \\
\text { Scheffe } \\
\text { 's post } \\
\text { hoc }\end{array}$ & $\begin{array}{l}\text { general } \\
\text { job } \\
\text { responsib } \\
\text { ilities } \\
\text { Mean } \pm S \\
D\end{array}$ & $\begin{array}{l}t / F / \\
\text { Scheff } \\
\text { e's } \\
\text { post } \\
\text { hoc }\end{array}$ & $\begin{array}{l}\text { nursing } \\
\text { care } \\
\text { responsibil } \\
\text { ities } \\
\text { Mean } \pm \text { SD }\end{array}$ & $\begin{array}{l}t / F / \\
\text { Scheff } \\
\text { e's } \\
\text { post } \\
\text { hoc }\end{array}$ & $\begin{array}{l}\text { relations } \\
\text { hips with } \\
\text { colleague } \\
\text { S } \\
\text { Mean } \pm \text { S } \\
\text { D }\end{array}$ & $\begin{array}{l}t / F / \\
\text { Scheff } \\
\text { e's } \\
\text { post } \\
\text { hoc }\end{array}$ & $\begin{array}{l}\text { relations } \\
\text { hips with } \\
\text { managem } \\
\text { ent } \\
\text { superviso } \\
\text { rs } \\
\text { Mean } \pm \text { S } \\
\text { D }\end{array}$ & $\begin{array}{l}t / F / \\
\text { Scheff } \\
\text { e's } \\
\text { post } \\
\text { hoc }\end{array}$ & $\begin{array}{l}\text { workloa } \\
\text { d and } \\
\text { work } \\
\text { procedu } \\
\text { res } \\
\text { Mean } \pm \text { S } \\
\text { D }\end{array}$ & $\begin{array}{l}t / F / \\
\text { Scheff } \\
\text { e's } \\
\text { post } \\
\text { hoc }\end{array}$ & $\begin{array}{l}\text { planning } \\
\text { and } \\
\text { sustainin } \\
\mathrm{g} \text { the } \\
\text { institutio } \\
\text { nal } \\
\text { environm } \\
\text { ent } \\
\text { Mean } \pm \mathrm{S} \\
\mathrm{D}\end{array}$ & $\begin{array}{l}t / F / \\
\text { Scheff } \\
\text { e's } \\
\text { post } \\
\text { hoc }\end{array}$ \\
\hline \multicolumn{16}{|l|}{ Gender } \\
\hline & 26 & $65.73 \pm 40$ & & $14.67 \pm 7$ & & $14.82 \pm 8.1$ & & $8.51 \pm 7.7$ & & $8.03 \pm 6.7$ & & $11.04 \pm 8$ & & $8.64 \pm 9.3$ & \\
\hline temale & 4 & 83 & & 15 & & 1 & & 9 & & 9 & & .94 & & 2 & \\
\hline Age & & & $4.37 * *$ & & $3.94 *$ & & 2.79 & & $3.30 *$ & & $4.47 * *$ & & $3.47 *$ & & $3.55 * *$ \\
\hline & 20 & $71.04 \pm 41$ & & $15.56 \pm 7$ & & $15.60 \pm 8.0$ & & $9.35 \pm 7.8$ & & $8.89 \pm 7.0$ & & $12.01 \pm 9$ & & $9.62 \pm 9.7$ & \\
\hline $21 \sim 30$ years & 0 & 94 & & 23 & & 4 & & 7 & & 8 & & .13 & & 4 & \\
\hline $31 \sim 55$ vears & 64 & $49.17 \pm 32$ & & $11.90 \pm 6$ & & $12.39 \pm 7.8$ & & $5.92 \pm 7.0$ & & $5.32 \pm 4.9$ & & $8.01 \pm 7$ & & $5.60 \pm 7.1$ & \\
\hline $31 \sim 55$ years & 64 & 20 & & 18 & & 7 & & 1 & & 4 & & 60 & & 4 & \\
\hline $\begin{array}{l}\text { Educational } \\
\text { level }\end{array}$ & & $21.29 * *$ & & $31.98 * *$ & & $18.89 * *$ & & & $\begin{array}{l}14.30 * \\
*\end{array}$ & & $\begin{array}{l}10.35 * \\
*\end{array}$ & & $\begin{array}{l}12.61 * \\
*\end{array}$ & & $\begin{array}{l}11.49 * \\
*\end{array}$ \\
\hline $\begin{array}{l}\text { 1.had } \\
\text { completed }\end{array}$ & & $60.14 \pm 45$ & $1<3$ & $11.84 \pm 5$ & $1<3$ & $13.32 \pm 8.1$ & $1<3$ & $8.25 \pm 8.3$ & & $7.65 \pm 6.9$ & & $10.02 \pm 1$ & $1<3$ & $9.02 \pm 10$ & \\
\hline $\begin{array}{l}\text { only middle } \\
\text { school }\end{array}$ & 70 & 80 & $2<3$ & 75 & $2<3$ & 9 & $2<3$ & 7 & $2<3$ & 7 & $2<3$ & 0.03 & $2<3$ & 99 & $<1,3$ \\
\hline 2.high school & 85 & $48.08 \pm 32$ & & $12.16 \pm 6$ & & $11.75 \pm 7.6$ & & $5.38 \pm 6.4$ & & $5.74 \pm 5.4$ & & $8.00 \pm$ & & $5.02 \pm$ & \\
\hline $\begin{array}{l}\text { or vocational } \\
\text { school }\end{array}$ & 85 & 04 & & 28 & & 8 & & 9 & & 8 & & 7.47 & & 6.72 & \\
\hline 3. college & 10 & $83.10 \pm 36$ & & $18.44 \pm 6$ & & $18.18 \pm 7.1$ & & $11.12 \pm 7$ & & $10.04 \pm 7$ & & $14.06 \pm 8$ & & $11.22 \pm$ & \\
\hline 3. college & 9 & 77 & & 94 & & 4 & & 47 & & 05 & & .35 & & 9.07 & \\
\hline $\begin{array}{l}\text { Years of } \\
\text { service }\end{array}$ & & & $-1.19 * *$ & & $2.42 * *$ & & -2.98 & & 0.14 & & 1.20 & & - & & - \\
\hline less than 2 & 12 & $62.65 \pm 44$ & & $13.59 \pm 6$ & & $13.32 \pm 8.2$ & & $8.58 \pm 7.8$ & & $8.54 \pm 7.1$ & & $10.17 \pm 9$ & & $8.42 \pm 10$ & \\
\hline years & 9 & 78 & & 41 & & 8 & & 6 & & 0 & & .78 & & 12 & \\
\hline 2 years or & 13 & $68.68 \pm 36$ & & $15.70 \pm 7$ & & $16.25 \pm 7.6$ & & $8.45 \pm 7.7$ & & $7.53 \pm 6.4$ & & $11.87 \pm 8$ & & $8.85 \pm$ & \\
\hline $\begin{array}{l}\text { above } \\
\text { Having } \\
\text { performed }\end{array}$ & 5 & 59 & & 67 & & 9 & & 6 & & 7 & & .00 & & 8.53 & \\
\hline $\begin{array}{l}\text { similar } \\
\text { nursing care } \\
\text { duties in } \\
\text { Taiwan }\end{array}$ & & & 2.57 & & 2.48 & & 2.43 & & 1.21 & & $2.41 * *$ & & 2.74 & & 1.74 \\
\hline & 10 & $73.79 \pm 39$ & & $16.03 \pm 6$ & & $16.34 \pm 7.5$ & & $9.25 \pm 7.9$ & & $9.33 \pm 7.3$ & & $12.92 \pm 8$ & & $9.90 \pm 9.3$ & \\
\hline yes & 2 & 09 & & 58 & & 3 & & 1 & & 5 & & .29 & & 3 & \\
\hline & 16 & $60.66 \pm 41$ & & $13.81 \pm 7$ & & $13.87 \pm 8.3$ & & $8.05 \pm 7.7$ & & $7.20 \pm 6.3$ & & $9.85 \pm 9$ & & $7.85 \pm 9.2$ & \\
\hline no & 2 & 21 & & 38 & & 3 & & 1 & & 1 & & 15 & & 6 & \\
\hline
\end{tabular}

Note: Using one-way ANOVA (Scheffe's post hoc); independent samples t-test, significant level $\alpha=.05$ two-tailed test; * $p<.05$ and $* * p<.01$

Table 1. Personal backgrounds of Vietnamese care attendants and analysis of the differences among various dimensions of occupational stress (continued; $N=264$ )

\begin{tabular}{|c|c|c|c|c|c|c|c|c|c|c|c|c|c|c|c|}
\hline Variables & $\mathbf{N}$ & $\begin{array}{l}\text { overall } \\
\text { occupatio } \\
\text { nal } \\
\text { stressor } \\
\text { Mean } \pm \text { SD }\end{array}$ & $\begin{array}{l}t / F / \\
\text { Scheff } \\
\text { e's } \\
\text { post } \\
\text { hoc }\end{array}$ & $\begin{array}{l}\text { general } \\
\text { job } \\
\text { responsi } \\
\text { bilities } \\
\text { Mean } \pm S \\
\text { D }\end{array}$ & $\begin{array}{l}t / F / \\
\text { Scheff } \\
\text { e's } \\
\text { post } \\
\text { hoc }\end{array}$ & $\begin{array}{l}\text { nursing } \\
\text { care } \\
\text { responsibi } \\
\text { lities } \\
\text { Mean } \pm \text { SD }\end{array}$ & $\begin{array}{l}t / F / \\
\text { Scheff } \\
\text { e's } \\
\text { post } \\
\text { hoc }\end{array}$ & $\begin{array}{l}\text { relations } \\
\text { hips } \\
\text { with } \\
\text { colleagu } \\
\text { es } \\
\text { Mean } \pm S \\
\text { D }\end{array}$ & $\begin{array}{l}t / F / \\
\text { Scheff } \\
\text { e's } \\
\text { post } \\
\text { hoc }\end{array}$ & $\begin{array}{l}\text { relations } \\
\text { hips } \\
\text { with } \\
\text { manage } \\
\text { ment } \\
\text { supervis } \\
\text { ors } \\
\text { Mean } \pm S \\
\text { D }\end{array}$ & $\begin{array}{l}t / F / \\
\text { Scheff } \\
\text { e's } \\
\text { post } \\
\text { hoc }\end{array}$ & $\begin{array}{l}\text { worklo } \\
\text { ad and } \\
\text { work } \\
\text { proced } \\
\text { ures } \\
\text { Mean } \pm \\
\text { SD }\end{array}$ & $\begin{array}{l}t / F / \\
\text { Scheff } \\
\text { e's } \\
\text { post } \\
\text { hoc }\end{array}$ & $\begin{array}{l}\text { planning } \\
\text { and } \\
\text { sustainin } \\
\text { g the } \\
\text { institutio } \\
\text { nal } \\
\text { environ } \\
\text { ment } \\
\text { Mean } \pm S \\
\text { D }\end{array}$ & $\begin{array}{l}t / F / \\
\text { Scheff } \\
\text { e's } \\
\text { post } \\
\text { hoc }\end{array}$ \\
\hline $\begin{array}{l}\text { The number of } \\
\text { non-work days } \\
\text { in a month }\end{array}$ & & & $\begin{array}{l}14.68 * \\
*\end{array}$ & & $\begin{array}{l}13.17 * \\
*\end{array}$ & $11.76^{* *}$ & & & 2.38 & & $6.04 * *$ & & $\begin{array}{l}20.55^{*} \\
*\end{array}$ & & $\begin{array}{l}13.94 * \\
*\end{array}$ \\
\hline 1.0 days & 13 & $\begin{array}{l}89.23 \pm 34 \\
83\end{array}$ & $3<1,2$ & $\begin{array}{l}17.38 \pm 7 \\
80\end{array}$ & $3<2$ & $\begin{array}{l}18.38 \pm 7.2 \\
6\end{array}$ & $3<2$ & $\begin{array}{l}11.69 \pm 9 . \\
43\end{array}$ & & $\begin{array}{l}8.84 \pm 4.8 \\
6\end{array}$ & $3<2$ & $\begin{array}{l}17.23 \pm 7 \\
.90\end{array}$ & $3<1,2$ & $\begin{array}{l}15.69 \pm 7 \\
85\end{array}$ & $3<1,2$ \\
\hline 2.1 3 days & 53 & $88.21 \pm 28$ & & $18.69 \pm 5$ & & $19.01 \pm 4.5$ & & $9.86 \pm 7.9$ & & $10.82 \pm 7$ & & $16.69 \pm 6$ & & $13.11 \pm 8$ & \\
\hline
\end{tabular}




\begin{tabular}{|c|c|c|c|c|c|c|c|c|c|c|c|c|c|c|c|}
\hline 3.4 12 days & $\begin{array}{l}19 \\
9\end{array}$ & $\begin{array}{l}26 \\
58.33 \pm 41 \text {. } \\
37\end{array}$ & & $\begin{array}{l}95 \\
13.44 \pm 6 . \\
99\end{array}$ & & $\begin{array}{l}5 \\
13.49 \pm 8.4 \\
6\end{array}$ & & $\begin{array}{l}9 \\
7.95 \pm 7.5 \\
8\end{array}$ & & $\begin{array}{l}76 \\
7.24 \pm 6.4 \\
5\end{array}$ & & $\begin{array}{l}.58 \\
9.16 \pm 8 . \\
76\end{array}$ & & $\begin{array}{l}21 \\
7.02 \pm 9.1 \\
2\end{array}$ & \\
\hline Lunch breaks & & & -7.46 & & -4.82 & & -4.42 & & -4.92 & & $-5.74 *$ & & -7.68 & & -8.36 \\
\hline yes & $\begin{array}{l}20 \\
6\end{array}$ & $\begin{array}{l}56.67 \pm 36 \\
67\end{array}$ & & $\begin{array}{l}13.59 \pm 6 . \\
94\end{array}$ & & $\begin{array}{l}13.69 \pm 7.9 \\
5\end{array}$ & & $\begin{array}{l}7.31 \pm 7.4 \\
1\end{array}$ & & $\begin{array}{l}6.68 \pm 5.9 \\
6\end{array}$ & & $\begin{array}{l}9.00 \pm 8 . \\
11\end{array}$ & & $\begin{array}{l}6.37 \pm 8.0 \\
8\end{array}$ & \\
\hline no & 58 & $\begin{array}{l}97.91 \pm 38 . \\
84\end{array}$ & & $\begin{array}{l}18.51 \pm 6 . \\
59\end{array}$ & & $\begin{array}{l}18.84 \pm 7.4 \\
2\end{array}$ & & $\begin{array}{l}12.79 \pm 7 . \\
67\end{array}$ & & $\begin{array}{l}12.79 \pm 7 . \\
44\end{array}$ & & $\begin{array}{l}18.25 \pm 8 \\
.01\end{array}$ & & $\begin{array}{l}16.70 \pm 9 . \\
05\end{array}$ & \\
\hline $\begin{array}{l}\text { Work hours in } \\
\text { a day }\end{array}$ & & & $\begin{array}{l}- \\
12.44 * \\
*\end{array}$ & & $\begin{array}{l}- \\
11.14 * \\
*\end{array}$ & & -12.60 & & $\begin{array}{l}- \\
7.85^{* *}\end{array}$ & & - & & $\begin{array}{l}- \\
11.90 * \\
*\end{array}$ & & $9.85 * *$ \\
\hline $8 \sim 10$ hours & $\begin{array}{l}11 \\
5\end{array}$ & $\begin{array}{l}37.75 \pm 30 . \\
16\end{array}$ & & $\begin{array}{l}10.20 \pm 4 . \\
78\end{array}$ & & $9.16 \pm 6.75$ & & $\begin{array}{l}6.79 \pm 5.5 \\
8\end{array}$ & & $\begin{array}{l}5.26 \pm 5.0 \\
5\end{array}$ & & $\begin{array}{l}5.08 \pm 6 . \\
73\end{array}$ & & $\begin{array}{l}3.24 \pm 7.0 \\
6\end{array}$ & \\
\hline $11 \sim 12$ hours & $\begin{array}{l}14 \\
9\end{array}$ & $\begin{array}{l}87.33 \pm 34 . \\
42\end{array}$ & & $\begin{array}{l}18.12 \pm 6 \\
76\end{array}$ & & $\begin{array}{l}19.19 \pm 6.1 \\
3\end{array}$ & & $\begin{array}{l}11.39 \pm 8 . \\
05\end{array}$ & & $\begin{array}{l}10.16 \pm 7 \\
20\end{array}$ & & $\begin{array}{l}15.63 \pm 7 \\
.64\end{array}$ & & $\begin{array}{l}12.81 \pm 8 . \\
72\end{array}$ & \\
\hline $\begin{array}{l}\text { Number of } \\
\text { patients cared } \\
\text { for during the } \\
\text { day }\end{array}$ & & & $\begin{array}{l}22.34 * \\
*\end{array}$ & & $\begin{array}{l}15.07 * \\
*\end{array}$ & & $\begin{array}{l}23.22 * \\
*\end{array}$ & & $\begin{array}{l}11.44 * \\
*\end{array}$ & & $\begin{array}{l}11.01^{*} \\
*\end{array}$ & & $\begin{array}{l}25.23 * \\
*\end{array}$ & & $\begin{array}{l}13.83^{*} \\
*\end{array}$ \\
\hline 1.6 10 patients & $\begin{array}{l}14 \\
1\end{array}$ & $\begin{array}{l}55.24 \pm 37 \text {. } \\
58\end{array}$ & $1<3$ & $\begin{array}{l}13.56 \pm 6 \\
34\end{array}$ & $1<3$ & $12.40 \pm 7.47$ & $71<2,3$ & $\begin{array}{l}7.70 \pm 7.3 \\
4\end{array}$ & $1<3$ & $\begin{array}{l}6.90 \pm 5.7 \\
1\end{array}$ & $1<3$ & $\begin{array}{l}8.09 \pm 8 . \\
18\end{array}$ & $1<2,3$ & $\begin{array}{l}6.56 \pm 8.7 \\
5\end{array}$ & $1<3$ \\
\hline $\begin{array}{l}2.11 \sim 15 \\
\text { patients }\end{array}$ & 88 & $\begin{array}{l}67.79 \pm 39 . \\
18\end{array}$ & $2<3$ & $\begin{array}{l}14.13 \pm 7 . \\
97\end{array}$ & $2<3$ & $\begin{array}{l}15.95 \pm 7.8 \\
3\end{array}$ & $2<3$ & $\begin{array}{l}7.57 \pm 7.9 \\
9\end{array}$ & $2<3$ & $\begin{array}{l}7.96 \pm 7.2 \\
0\end{array}$ & $2<3$ & $\begin{array}{l}12.81 \pm 8 \\
.15\end{array}$ & $2<3$ & $\begin{array}{l}9.34 \pm 8.6 \\
9\end{array}$ & $2<3$ \\
\hline 3. $\geqq 16$ patients & 35 & $\begin{array}{l}102.85 \pm 35 \\
.60\end{array}$ & & $\begin{array}{l}20.51 \pm 5 . \\
15\end{array}$ & & $\begin{array}{l}21.74 \pm 6.6 \\
7\end{array}$ & & $\begin{array}{l}14.17 \pm 6 . \\
84\end{array}$ & & $\begin{array}{l}12.71 \pm 7 . \\
91\end{array}$ & & $\begin{array}{l}18.42 \pm 8 \\
.53\end{array}$ & & $\begin{array}{l}15.28 \pm 9 . \\
96\end{array}$ & \\
\hline $\begin{array}{l}\text { Number of } \\
\text { patients cared } \\
\text { for during the } \\
\text { night }\end{array}$ & & $39.07 * *$ & & & $\begin{array}{l}31.31 * \\
*\end{array}$ & & $\begin{array}{l}49.05 * \\
*\end{array}$ & & $\begin{array}{l}16.71 * \\
*\end{array}$ & & $9.60 * *$ & & $\begin{array}{l}38.43 * \\
*\end{array}$ & & $\begin{array}{l}25.91 * \\
*\end{array}$ \\
\hline 1.6 10 patients & 91 & $\begin{array}{l}39.17 \pm 28 . \\
24\end{array}$ & $1<2,3$ & $\begin{array}{l}10.37 \pm 4 . \\
41\end{array}$ & $1<2,3$ & $9.54 \pm 6.58$ & $1<2,3$ & $\begin{array}{l}4.93 \pm 5.5 \\
8\end{array}$ & $1<2,3$ & $\begin{array}{l}5.59 \pm 4.8 \\
0\end{array}$ & $1<2,3$ & $\begin{array}{l}5.29 \pm 6 . \\
32\end{array}$ & $1<2,3$ & $\begin{array}{l}3.42 \pm 6.6 \\
3\end{array}$ & $1<2,3$ \\
\hline $\begin{array}{l}2.11 \sim 15 \\
\text { patients }\end{array}$ & 96 & $\begin{array}{l}75.92 \pm 42 . \\
41\end{array}$ & & $\begin{array}{l}16.52 \pm 7 \\
46\end{array}$ & & $\begin{array}{l}15.60 \pm 7.9 \\
0\end{array}$ & $2<3$ & $\begin{array}{l}10.02 \pm 7 \\
96\end{array}$ & & $\begin{array}{l}9.51 \pm 7.2 \\
1\end{array}$ & & $\begin{array}{l}13.09 \pm 8 \\
.98\end{array}$ & & $\begin{array}{l}11.17 \pm 9 \\
85\end{array}$ & \\
\hline $3 . \geqq 16$ patients & 77 & $\begin{array}{l}84.42 \pm 35 \text {. } \\
32\end{array}$ & & $\begin{array}{l}17.45 \pm 7 . \\
08\end{array}$ & & $\begin{array}{l}20.09 \pm 6.0 \\
0\end{array}$ & & $\begin{array}{l}10.88 \pm 8 \\
38\end{array}$ & & $\begin{array}{l}9.06 \pm 7.5 \\
1\end{array}$ & & $\begin{array}{l}15.27 \pm 8 \\
.09\end{array}$ & & $\begin{array}{l}11.66 \pm 8 \\
81\end{array}$ & \\
\hline
\end{tabular}

Note: Using one-way ANOVA (Scheffe's post hoc); independent samples t-test, significant level $\alpha=.05$ two-tailed test; ${ }^{*} p<.05$ and ${ }^{* *} p<.01$

Table 1. Personal backgrounds of Vietnamese care attendants and analysis of the differences among various dimensions of occupational stress (continued; $N=264)$

\begin{tabular}{|c|c|c|c|c|c|c|c|c|c|c|c|c|c|c|c|}
\hline Variables & $\mathbf{N}$ & $\begin{array}{l}\text { overall } \\
\text { occupatio } \\
\text { nal } \\
\text { stressor } \\
\text { Mean } \pm \text { SD }\end{array}$ & $\begin{array}{l}t / F / \\
\text { Scheff } \\
\text { e's } \\
\text { post } \\
\text { hoc }\end{array}$ & $\begin{array}{l}\text { general } \\
\text { job } \\
\text { responsi } \\
\text { bilities } \\
\text { Mean } \pm S \\
\text { D }\end{array}$ & $\begin{array}{l}t / F / \\
\text { Scheff } \\
\text { e's } \\
\text { post } \\
\text { hoc }\end{array}$ & $\begin{array}{l}\text { nursing } \\
\text { care } \\
\text { responsib } \\
\text { ilities } \\
\text { Mean } \pm \text { SD }\end{array}$ & $\begin{array}{l}t / F / \\
\text { Scheff } \\
\text { e's } \\
\text { post } \\
\text { hoc }\end{array}$ & $\begin{array}{l}\text { relation } \\
\text { ships } \\
\text { with } \\
\text { colleagu } \\
\text { es } \\
\text { Mean } \pm S \\
\text { D }\end{array}$ & $\begin{array}{l}t / F / \\
\text { Scheff } \\
\text { e's } \\
\text { post } \\
\text { hoc }\end{array}$ & $\begin{array}{l}\text { relation } \\
\text { ships } \\
\text { with } \\
\text { manage } \\
\text { ment } \\
\text { supervis } \\
\text { ors } \\
\text { Mean } \pm S \\
\text { D }\end{array}$ & $\begin{array}{l}t / F / \\
\text { Scheff } \\
\text { e's } \\
\text { post } \\
\text { hoc }\end{array}$ & $\begin{array}{l}\text { workloa } \\
\text { d and } \\
\text { work } \\
\text { procedur } \\
\text { es } \\
\text { Mean } \pm S \\
\text { D }\end{array}$ & $\begin{array}{l}t / F / \\
\text { Scheff } \\
\text { e's } \\
\text { post } \\
\text { hoc }\end{array}$ & $\begin{array}{l}\text { planning } \\
\text { and } \\
\text { sustainin } \\
\text { g the } \\
\text { institutio } \\
\text { nal } \\
\text { environ } \\
\text { ment } \\
\text { Mean } \pm S \\
\text { D }\end{array}$ & $\begin{array}{l}t / F / \\
\text { Scheff } \\
\text { e's } \\
\text { post } \\
\text { hoc }\end{array}$ \\
\hline $\begin{array}{l}\text { Received } \\
\text { nursing care } \\
\text { training } \\
\text { courses in } \\
\text { Vietnam }\end{array}$ & & & -5.29 & & -2.00 & -3.70 & & & -6.21 & & $-3.42 *$ & & -4.84 & & -5.20 \\
\hline yes & $\begin{array}{l}24 \\
8 \\
16\end{array}$ & $\begin{array}{l}62.52 \pm 38 . \\
64 \\
115.62 \pm 42 \\
.67\end{array}$ & & $\begin{array}{l}14.45 \pm 7 \\
18 \\
18.12 \pm 5 \\
81\end{array}$ & & $\begin{array}{l}14.36 \pm 7.8 \\
9 \\
21.93 \pm 8.2 \\
9\end{array}$ & & $\begin{array}{l}7.81 \pm 7.3 \\
5 \\
19.50 \pm 6 . \\
21\end{array}$ & & $\begin{array}{l}7.57 \pm 6.4 \\
2 \\
15.06 \pm 8 . \\
59\end{array}$ & & $\begin{array}{l}10.39 \pm 8 \\
59 \\
21.12 \pm 8 \\
46\end{array}$ & & $\begin{array}{l}7.92 \pm \\
8.79 \\
19.87 \pm 10 \\
.46\end{array}$ & \\
\hline $\begin{array}{l}\text { On-the-job } \\
\text { training in } \\
\text { Taiwan }\end{array}$ & & & $2.33^{*}$ & & 2.59 & & $2.00 *$ & & $\begin{array}{l}1.81 * \\
*\end{array}$ & & $0.85 * *$ & & 3.19 & & 1.60 \\
\hline yes & $\begin{array}{l}10 \\
1 \\
16 \\
3\end{array}$ & $\begin{array}{l}73.46 \pm 45 . \\
40 \\
60.95 \pm 37 . \\
07\end{array}$ & & $\begin{array}{l}16.10 \pm 7 . \\
39 \\
13.78 \pm 6 . \\
87\end{array}$ & & $\begin{array}{l}16.13 \pm 8.8 \\
1 \\
14.01 \pm 7.5 \\
5\end{array}$ & & $\begin{array}{l}9.66 \pm 8.5 \\
9 \\
7.80 \pm 7.2 \\
0\end{array}$ & & $\begin{array}{l}8.50 \pm 7.7 \\
3 \\
7.73 \pm 6.1 \\
5\end{array}$ & & $\begin{array}{l}13.23 \pm 9 . \\
36 \\
9.68 \pm 8.4 \\
1\end{array}$ & & $\begin{array}{l}9.81 \pm 10 . \\
14 \\
7.92 \pm \\
8.74\end{array}$ & \\
\hline $\begin{array}{l}\text { Place of } \\
\text { residence }\end{array}$ & & & -4.33 & & -1.78 & & - $7.54 *$ & & -4.81 & & -2.29 & & -3.6 & & -3.18 \\
\hline
\end{tabular}




\begin{tabular}{|c|c|c|c|c|c|c|c|c|c|c|c|c|c|c|c|}
\hline & & & & & & & * & & & & & & & & \\
\hline \multirow{5}{*}{$\begin{array}{l}\text { assigned a } \\
\text { room by the } \\
\text { institution } \\
\text { shelter next to } \\
\text { patients } \\
\text { Living } \\
\text { environment }\end{array}$} & 23 & $61.68 \pm 39$ & & $14.37 \pm 7$ & & $13.72 \pm 7.8$ & & $7.66 \pm 7.3$ & & $7.66 \pm 6.6$ & & $10.29 \pm 8$ & & $9.95 \pm$ & \\
\hline & 0 & 79 & & 28 & & 1 & & 1 & & 5 & & 76 & & 8.97 & \\
\hline & 34 & $93.17 \pm 37$ & & $16.70 \pm 5$ & & $22.26 \pm 5.8$ & & $14.29 \pm 8$ & & $10.50 \pm 7$ & & $16.08 \pm 8$ & & $13.32 \pm 10$ & \\
\hline & 34 & 56 & & 86 & & 7 & & 58 & & 32 & & 64 & & .44 & \\
\hline & & & 7.63 & & 5.37 & & 6.18 & & 4.95 & & $4.23 *$ & & 7.38 & & 8.55 \\
\hline loud and & 38 & $108.13 \pm 35$ & & $20.15 \pm 5$ & & $21.86 \pm 6.4$ & & $14.07 \pm 8$ & & $12.68 \pm 7$ & & $20.07 \pm 6$ & & $19.26 \pm 7$ & \\
\hline unclean & 38 & .39 & & 51 & & 3 & & 82 & & 46 & & 88 & & 74 & \\
\hline neat and cloan & 22 & $58.61 \pm 37$ & & $13.75 \pm 6$ & & $13.64 \pm 7.7$ & & $7.58 \pm 7.2$ & & $7.24 \pm 6.3$ & & $9.52 \pm 8.3$ & & $6.86 \pm 8.3$ & \\
\hline neat and clean & 6 & 26 & & 99 & & 6 & & 2 & & 7 & & 4 & & 4 & \\
\hline
\end{tabular}

Note: Using one-way ANOVA (Scheffe's post hoc); independent samples t-test, significant level $\alpha=.05$ two-tailed test; $* p<.05$ and $* * p<.01$

\subsection{Relationship between Personal Backgrounds and Quality of Life of Vietnamese Care Attendants}

The QOL was higher for older age groups than for younger age groups, and was higher for those who had a high school and vocational school education than for those with a middle school or college education. The QOL peaked when the number of non-work days in a month was between 4 and 12. The results also showed that the QOL was higher when the number of daily work hours was between 8 and 10, rather than between 11 and 12, and was higher when the number of patients cared for during the day and night was 6-10,rather than 11-15 or more than 16 . In addition, the PCS and MCS differed significantly for different years of service those with less than two years of service fared better than those working for more than two years. The QOL for MCS was higher for those who had not performed similar nursing care duties in Taiwan than for those who had, and was higher for those who had received nursing care training courses in Vietnam than for those who had not (Table 2).

Table 2. Analysis of the differences in $Q O L$ and personal backgrounds of Vietnamese care attendants $(N=264)$

\begin{tabular}{|c|c|c|c|c|c|c|c|c|c|c|c|c|c|c|c|}
\hline Variables & $\mathbf{N}$ & $\begin{array}{l}\text { SF-36 } \\
\text { Mean } \pm \\
\text { SD }\end{array}$ & $\begin{array}{l}t / F / \\
\text { Scheffe' } \\
\text { s post } \\
\text { hoc }\end{array}$ & $\begin{array}{l}\text { PCS } \\
\text { Mean } \pm \mathbf{S} \\
\text { D }\end{array}$ & $\begin{array}{l}t / F / \\
\text { Scheffe' } \\
\text { s post } \\
\text { hoc }\end{array}$ & $\begin{array}{l}\text { MCS } \\
\text { Mean } \pm \\
\text { SD }\end{array}$ & $\begin{array}{l}t / F / \\
\text { Scheff } \\
\text { e's } \\
\text { post } \\
\text { hoc } \\
\end{array}$ & Variables & $\mathbf{N}$ & $\begin{array}{l}\text { SF-36 } \\
\text { Mean } \pm \\
\text { SD }\end{array}$ & $\begin{array}{l}t / F / \\
\text { Scheff } \\
\text { e's } \\
\text { post } \\
\text { hoc } \\
\end{array}$ & $\begin{array}{l}\text { PCS } \\
\text { Mean } \pm S \\
\text { D }\end{array}$ & $\begin{array}{l}t / F / \\
\text { Scheff } \\
\text { e's } \\
\text { post } \\
\text { hoc } \\
\end{array}$ & $\begin{array}{l}\text { MCS } \\
\text { Mean } \pm S \\
\text { D }\end{array}$ & $\begin{array}{l}t / F / \\
\text { Scheff } \\
\text { e's } \\
\text { post } \\
\text { hoc } \\
\end{array}$ \\
\hline Age & & & $-3.44 * *$ & & $-3.22 * *$ & & $3.15 * *$ & $\begin{array}{l}\text { Number of } \\
\text { patients cared } \\
\text { for during the } \\
\text { night }\end{array}$ & & & $\begin{array}{l}19.33 * \\
*\end{array}$ & & $\begin{array}{l}14.97 * \\
*\end{array}$ & & $\begin{array}{l}18.76^{*} \\
*\end{array}$ \\
\hline 21 30 years & $\begin{array}{l}20 \\
0\end{array}$ & $\begin{array}{l}70.38 \pm 1 \\
4.43\end{array}$ & & $\begin{array}{l}70.69 \pm 1 \\
6.30\end{array}$ & & $\begin{array}{l}70.07 \pm 1 \\
4.63\end{array}$ & & 1.6 10 patients & 91 & $\begin{array}{l}78.48 \pm 1 \\
1.29\end{array}$ & $2<1$ & $\begin{array}{l}78.90 \pm 12 \\
.80\end{array}$ & $2<1$ & $\begin{array}{l}78.05 \pm 10 \\
.82\end{array}$ & $2<1$ \\
\hline $31 \sim 55$ years & 64 & $\begin{array}{l}76.07 \pm 1 \\
0.39\end{array}$ & & $\begin{array}{l}76.92 \pm 1 \\
2.38\end{array}$ & & $\begin{array}{l}75.23 \pm 1 \\
0.10\end{array}$ & & $\begin{array}{l}2.11 \sim 15 \\
\text { patients }\end{array}$ & 96 & $\begin{array}{l}69.11 \pm 1 \\
4.87\end{array}$ & $3<1$ & $\begin{array}{l}70.04 \pm 16 \\
.51\end{array}$ & $3<1$ & $\begin{array}{l}68.17 \pm 15 \\
.40\end{array}$ & $3<1$ \\
\hline $\begin{array}{l}\text { Educational } \\
\text { level }\end{array}$ & & & $19.16^{* *}$ & & $18.97 * *$ & & $\begin{array}{l}14.18 * \\
*\end{array}$ & $3 . \geqq 16$ patients & 77 & $\begin{array}{l}67.13 \pm 1 \\
1.97\end{array}$ & & $\begin{array}{l}66.98 \pm 15 \\
.01\end{array}$ & & $\begin{array}{l}67.29 \pm 11 \\
.98\end{array}$ & \\
\hline $\begin{array}{l}\text { 1.had } \\
\text { completed } \\
\text { only middle } \\
\text { school }\end{array}$ & 70 & $\begin{array}{l}73.73 \pm 1 \\
2.22\end{array}$ & $3<1,2$ & $\begin{array}{l}75.28 \pm 1 \\
4.32\end{array}$ & $3<1,2$ & $\begin{array}{l}72.19 \pm 1 \\
1.30\end{array}$ & $3<1,2$ & Lunch breaks & & & 7.64 & & 7.38 & & 6.64 \\
\hline $\begin{array}{l}\text { 2.high school } \\
\text { or vocational } \\
\text { school }\end{array}$ & 85 & $\begin{array}{l}77.35 \pm 1 \\
2.35\end{array}$ & & $\begin{array}{l}78.02 \pm 1 \\
3.23\end{array}$ & & $\begin{array}{l}76.68 \pm 1 \\
3.59\end{array}$ & & yes & $\begin{array}{l}20 \\
6\end{array}$ & $\begin{array}{l}74.87 \pm 1 \\
2.60\end{array}$ & & $\begin{array}{l}75.64 \pm 14 \\
.10\end{array}$ & & $\begin{array}{l}74.10 \pm 12 \\
.95\end{array}$ & \\
\hline 3. college & $\begin{array}{l}10 \\
9\end{array}$ & $\begin{array}{l}66.13 \pm 1 \\
3.71\end{array}$ & & $\begin{array}{l}65.68 \pm 1 \\
5.92\end{array}$ & & $\begin{array}{l}66.59 \pm 1 \\
3.96\end{array}$ & & no & 58 & $\begin{array}{l}60.71 \pm 1 \\
1.98\end{array}$ & & $\begin{array}{l}59.97 \pm 14 \\
.84\end{array}$ & & $\begin{array}{l}61.44 \pm 12 \\
.33\end{array}$ & \\
\hline $\begin{array}{l}\text { Years of } \\
\text { service }\end{array}$ & & & 1.58 & & $2.60 *$ & & $0.23 *$ & $\begin{array}{l}\text { Work hours in } \\
\text { a day }\end{array}$ & & & $\begin{array}{l}10.41 * \\
*\end{array}$ & & $\begin{array}{l}10.60 * \\
*\end{array}$ & & $8.38 * *$ \\
\hline $\begin{array}{l}\text { less than } 2 \\
\text { years }\end{array}$ & $\begin{array}{l}12 \\
9\end{array}$ & $\begin{array}{l}73.13 \pm 1 \\
3.68\end{array}$ & & $\begin{array}{l}74.73 \pm 1 \\
4.07\end{array}$ & & $\begin{array}{l}71.52 \pm 1 \\
4.68\end{array}$ & & $8 \sim 10$ hours & $\begin{array}{l}11 \\
5\end{array}$ & $\begin{array}{l}80.03 \pm \\
9.99\end{array}$ & & $\begin{array}{l}81.65 \pm 10 \\
.59\end{array}$ & & $\begin{array}{l}78.41 \pm 10 \\
.97\end{array}$ & \\
\hline $\begin{array}{l}2 \text { years or } \\
\text { above }\end{array}$ & $\begin{array}{l}13 \\
5\end{array}$ & $\begin{array}{l}70.45 \pm 1 \\
3.76\end{array}$ & & $\begin{array}{l}69.78 \pm 1 \\
6.72\end{array}$ & & $\begin{array}{l}71.13 \pm 1 \\
3.02\end{array}$ & & $11 \sim 12$ hours & $\begin{array}{l}14 \\
9\end{array}$ & $\begin{array}{l}65.38 \pm 1 \\
2.86\end{array}$ & & $\begin{array}{l}64.90 \pm 15 \\
.03\end{array}$ & & $\begin{array}{l}65.85 \pm 13 \\
.34\end{array}$ & \\
\hline $\begin{array}{l}\text { Having } \\
\text { performed } \\
\text { similar } \\
\text { nursing care } \\
\text { duties in } \\
\text { Taiwan }\end{array}$ & & & -2.15 & & -1.14 & & - $2.87 * *$ & $\begin{array}{l}\text { Received } \\
\text { nursing care } \\
\text { training } \\
\text { courses in } \\
\text { Vietnam }\end{array}$ & & & 1.96 & & 1.25 & & $4.59 * *$ \\
\hline yes & $\begin{array}{l}10 \\
2 \\
16\end{array}$ & $\begin{array}{l}69.48 \pm 1 \\
4.16 \\
73.20 \pm 1\end{array}$ & & $\begin{array}{l}70.82 \pm 1 \\
5.01 \\
73.07 \pm 1\end{array}$ & & $\begin{array}{l}68.14 \pm 1 \\
5.23 \\
73.32 \pm 1\end{array}$ & & $\begin{array}{l}\text { yes } \\
\text { no }\end{array}$ & $\begin{array}{l}24 \\
8 \\
16\end{array}$ & $\begin{array}{l}72.18 \pm 1 \\
3.77 \\
65.24 \pm 1\end{array}$ & & $\begin{array}{l}72.51 \pm 15 \\
.30 \\
67.43 \pm 20\end{array}$ & & $\begin{array}{l}71.85 \pm 14 \\
.00 \\
63.04 \pm\end{array}$ & \\
\hline
\end{tabular}




\begin{tabular}{|c|c|c|c|c|c|c|c|c|c|c|c|c|c|c|c|}
\hline & 2 & 3.35 & & 6.08 & & 2.51 & & & & 2.14 & & .34 & & 6.79 & \\
\hline $\begin{array}{l}\text { The number } \\
\text { of non-work } \\
\text { days in a } \\
\text { month }\end{array}$ & & & $14.10^{* *}$ & & $17.40 * *$ & & $7.55 * *$ & $\begin{array}{l}\text { On-the-job } \\
\text { training in } \\
\text { Taiwan }\end{array}$ & & & 0.07 & & 0.13 & & 0.00 \\
\hline 1.0 days & 13 & $\begin{array}{l}62.93 \pm 1 \\
4.29\end{array}$ & $1<3$ & $\begin{array}{l}60.48 \pm 1 \\
6.86\end{array}$ & $1<3$ & $\begin{array}{l}65.38 \pm 1 \\
3.41\end{array}$ & $2<3$ & yes & $\begin{array}{l}10 \\
1\end{array}$ & $\begin{array}{l}71.84 \pm 1 \\
3.33\end{array}$ & & $\begin{array}{l}72.36 \pm 15 \\
.02\end{array}$ & & $\begin{array}{l}71.32 \pm 13 \\
.36\end{array}$ & \\
\hline 2.1 3 days & 53 & $\begin{array}{l}64.61 \pm 1 \\
2.94\end{array}$ & $2<3$ & $\begin{array}{l}63.48 \pm 1 \\
5.56\end{array}$ & $2<3$ & $\begin{array}{l}65.75 \pm 1 \\
3.88\end{array}$ & & no & $\begin{array}{l}16 \\
3\end{array}$ & $\begin{array}{l}71.71 \pm 1 \\
4.06\end{array}$ & & $\begin{array}{l}72.10 \pm 16 \\
.07\end{array}$ & & $\begin{array}{l}71.32 \pm 14 \\
.55\end{array}$ & \\
\hline 3.4 12 days & $\begin{array}{l}19 \\
9\end{array}$ & $\begin{array}{l}74.20 \pm 1 \\
3.09\end{array}$ & & $\begin{array}{l}75.24 \pm 1 \\
4.40\end{array}$ & & $\begin{array}{l}73.17 \pm 1 \\
3.40\end{array}$ & & $\begin{array}{l}\text { Place of } \\
\text { residence }\end{array}$ & & & 2.28 & & 1.97 & & 2.29 \\
\hline $\begin{array}{l}\text { Number of } \\
\text { patients } \\
\text { cared for } \\
\text { during the } \\
\text { day }\end{array}$ & & & $15.09 * *$ & & $12.90 * *$ & & $\begin{array}{l}13.27 * \\
*\end{array}$ & $\begin{array}{l}\text { assigned a room } \\
\text { by the } \\
\text { institution }\end{array}$ & $\begin{array}{l}23 \\
0\end{array}$ & $\begin{array}{l}72.50 \pm 1 \\
3.88\end{array}$ & & $\begin{array}{l}72.93 \pm 15 \\
.74\end{array}$ & & $\begin{array}{l}72.07 \pm 13 \\
.80\end{array}$ & \\
\hline $\begin{array}{l}1.6 \sim 10 \\
\text { patients }\end{array}$ & $\begin{array}{l}14 \\
1\end{array}$ & $\begin{array}{l}74.82 \pm 1 \\
3.20\end{array}$ & $3<1,2$ & $\begin{array}{l}75.68 \pm 1 \\
5.16\end{array}$ & $3<1,2$ & $\begin{array}{l}73.95 \pm 1 \\
2.51\end{array}$ & $3<1,2$ & $\begin{array}{l}\text { shelter next to } \\
\text { patients }\end{array}$ & 34 & $\begin{array}{l}66.77 \pm 1 \\
1.90\end{array}$ & & $\begin{array}{l}67.27 \pm 14 \\
.28\end{array}$ & & $\begin{array}{l}66.28 \pm 13 \\
.15\end{array}$ & \\
\hline $\begin{array}{l}2.11 \sim 15 \\
\text { patients }\end{array}$ & 88 & $\begin{array}{l}71.01 \pm 1 \\
3.10\end{array}$ & & $\begin{array}{l}70.84 \pm 1 \\
4.35\end{array}$ & & $\begin{array}{l}71.17 \pm 1 \\
4.58\end{array}$ & & $\begin{array}{l}\text { Living } \\
\text { environment }\end{array}$ & & & -5.15 & & -4.29 & & -5.35 \\
\hline $\begin{array}{l}3 . \geqq 16 \\
\text { patients }\end{array}$ & 35 & $\begin{array}{l}61.34 \pm 1 \\
2.48\end{array}$ & & $\begin{array}{l}61.60 \pm 1 \\
5.84\end{array}$ & & $\begin{array}{l}61.08 \pm 1 \\
2.41\end{array}$ & & $\begin{array}{l}\text { loud and } \\
\text { unclean }\end{array}$ & 38 & $\begin{array}{l}61.58 \pm 1 \\
1.24\end{array}$ & & $\begin{array}{l}64.43 \pm 13 \\
.65\end{array}$ & & $\begin{array}{l}60.74 \pm 11 \\
.52\end{array}$ & \\
\hline
\end{tabular}

Note: Using one-way ANOVA (Scheffe's post hoc); independent samples t-test, significant level $\alpha=.05$ two-tailed test; ${ }^{*} p<.05$ and $* * p<.01$

\subsection{The Factors Influencing Quality of Life Major Predictors of QOL}

Results from stepwise multiple regression analysis showed that the variables "workload and work procedures," "number of daily work hours," and "relationships with management supervisors" in the regression model could predict QOL for SF-36, PCS, and MCS, with a respective variance of $44.9 \%, 38.3 \%$, and $41.7 \%$. A one-point increase in "workload and work procedures" resulted in decreases of $0.440,0.409$, and 0.472 in the three QOL scores. An 11-12hour work schedule, compared to an 8-10-hour work schedule, resulted in decreases of 7.697, 10.548, and 4.846 in the three QOL scores. A one-point increase in "stressful relationships with management supervisors" resulted in decreases of $0.473,0.385$, and 0.560 in the three QOL scores (Table 3 ).

Table 3. Stepwise multiple regression analysis of the effects of personal background and various dimensions of occupational stress on the QOL of Vietnamese care attendants $(N=264)$

\begin{tabular}{|c|c|c|c|c|c|c|}
\hline $\begin{array}{l}\text { Variables } \\
\text { Modelone }\end{array}$ & B & Beta & $R^{2}$ & $\Delta \mathbf{R}^{2}$ & $\mathbf{t}$ & $\mathbf{F}$ \\
\hline \multicolumn{7}{|l|}{$\underline{\text { SF-36 Impact factor }}$} \\
\hline constant & 92.463 & & & & $42.001^{* * *}$ & \\
\hline workload and work procedures & -0.440 & -0.286 & 0.381 & 0.381 & $-3.716^{* * *}$ & $161.052^{* * *}$ \\
\hline the number of daily work hours ${ }^{\mathrm{a}}$ & -7.697 & -0.278 & 0.423 & 0.043 & $-4.858^{* * *}$ & $95.795^{* * *}$ \\
\hline relationships with colleagues & -0.473 & -0.233 & 0.449 & 0.026 & $-3.495^{* * *}$ & $70.681^{* * *}$ \\
\hline \multicolumn{7}{|l|}{ Modeltwo } \\
\hline \multicolumn{7}{|l|}{$\underline{\text { PCS Impact factor }}$} \\
\hline constant & 96.314 & & & & $36.333^{* * *}$ & \\
\hline workload and work procedures & -0.409 & -0.234 & 0.303 & 0.303 & $-2.867^{* *}$ & $113.784^{* * *}$ \\
\hline the number of daily work hours ${ }^{\mathrm{a}}$ & -10.548 & -0.335 & 0.369 & 0.067 & $-5.529^{* * *}$ & $76.411^{* * *}$ \\
\hline relationships with colleagues & -0.385 & -0.167 & 0.383 & 0.013 & $-2.365^{* *}$ & $53.701^{* * *}$ \\
\hline \multicolumn{7}{|l|}{ Modelthree } \\
\hline \multicolumn{7}{|l|}{ MCS Impact factor } \\
\hline constant & 88.611 & & & & $38.905^{* * *}$ & \\
\hline workload and work procedures & -0.472 & -0.305 & 0.366 & 0.366 & $-3.847^{* * *}$ & $151.348^{* * *}$ \\
\hline relationships with colleagues & -0.560 & -0.275 & 0.397 & 0.031 & $-4.005^{* * *}$ & $85.813^{* * *}$ \\
\hline the number of daily work hours ${ }^{\mathrm{a}}$ & -4.846 & -0.174 & 0.417 & 0.020 & $-2.956^{* *}$ & $61.817^{* * *}$ \\
\hline
\end{tabular}

Note: 1. Variables adjusted include personal background (educational level, number of non-work days in a month, the number of daily work hours, most patients cared for during the day, most patients cared for at night), general job responsibilities, nursing care responsibilities, relationships with colleagues, workload and work procedures, and sustaining the institutional environment.

2. $* * p<.01 \quad * * * p<.001$. a: $8-10$ hours $=0 ; 11-12$ hours $=1$ 


\section{Discussion}

This is the first study to examine the effects of personal background and occupational stress on the QOL of Vietnamese care attendants working at medical institutions in Taiwan. With the rapid increase in the elderly population in Taiwan, the demand for nurses at medical institutions has grown substantially. Therefore, a closer understanding of the factors that influence the QOL of these Vietnamese care attendants may effectively elevate the QOL of care attendants and the quality of nursing care for the patients.

This study showed that younger care attendants experienced higher levels of occupational stress, as found in similar research $[20,21]$. Those who graduated from college experienced greater occupational stress than did those from middle, high, and vocational schools because of the higher job expectations and aspirations, which corresponded closely to the results of Lin (2000) [9]. Furthermore, those working two years or more experienced more occupational stress than did their counterparts. Those who had performed similar nursing care duties in Taiwan, had not received nursing care training courses in Vietnam, and had had on-the-job training in Taiwan experienced higher levels of occupational stress. Those who worked 1112 hours a day experienced greater occupational stress than did those working 8-10 hours. Those working longer shifts generally experienced more occupational stress. These results are comparable to those obtained from previous research $[4,8,9,20]$. In this study, the Vietnamese care attendants provided an average of 10.58 hours of nursing care daily, and the long work hours easily transformed into an additional load on the body. Those working without lunch breaks, having fewer non-work days in a month, and who cared for more than 16 patients experienced higher levels of occupational stress, as did those who took shelter next to patients and lived in loud and unclean environments. Living in affixed compartments built by the institution or beside the patients also created higher levels of occupational stress because of the lack of space, poor soundproofing, and noise from open spaces. These results are comparable to those of previous research $[6,8,9]$.

This study showed that, for Vietnamese care attendants, QOL dimension variables such as SF-36, PCS, and MCS differed significantly for those in different age groups, and with different educational levels, different numbers of nonwork days in a month, different numbers of daily work hours, and different numbers of patients cared for during the day and night, similar to the results of other foreign and domestic research $[6,17,22,23]$. Those with higher educational levels often experienced higher QOL, and those with fewer patients to care for, who had received complete on-the-job training in Taiwan and who clearly understood the work content were able to reduce the time for nursing care, thereby allowing themselves adequate breaks and vacation time to rest. If the scheduling of vacations were available, it would help to relieve tension and allow the body to recover, thus producing higher QOL [24-26].

The results from the stepwise multiple regression analysis showed that the variables "workload and work procedures," "the number of daily work hours," and "relationships with management supervisors" could be used to predict the QOL for SF-36, PCS, and MCS with a respective variance of $44.9 \%, 38.3 \%$, and $41.7 \%$. Regarding "workload and work procedures" and "number of daily work hours," the most common causes of occupational stress was "requirement to do heavy work," followed by "inability to rest at will," similar to the results of previous research[7,27]. Institutions should consider the ability of the Vietnamese care attendants and the workload being given to them. By effectively reducing their workload and teaching them the correct work methodology, medical institutions will be able to provide nursing care in a less time-consuming and more efficient manner. In "relationships with management supervisors," it was found that the management approaches of medical institution supervisors have often seemed condescending to Vietnamese care attendants, and Vietnamese care attendants' relationships with management supervisors have often deteriorated because of language barriers; these results corresponded to those of previous research [4]. It is highly recommended that management supervisors be more sympathetic toward their subordinates, and learn to interact and communicate as a way to understand their needs.

"Workload and work procedures" was observed to have the greatest impact on the QOL of Vietnamese care attendants. This result contradicts that of the research on foreign care attendants working in nursing homes, which identified "relationships with management supervisors" as having the greatest impact [6]. This result may be due to our research target being Vietnamese care attendants working at a medical institution instead of foreign care attendants working in a nursing home. This finding was not derived by accounting for the complexity of hospital work or the numerous rules, regulations, and standard operational procedures involved. Vietnamese care attendants working away from home must not only cope with the difficulty of trying to communicate, but also overcome the difficulties of complex and strenuous work, which creates additional levels of occupational stress that continue to affect their QOL.

The present study bears the limitations of a crosssectional study design, which makes causal inference less certain. In addition, the results of this research were based on the study of Vietnamese care attendants working in Kaohsiung City in southern Taiwan. The possibility of selection bias could not be completely avoided. As a regional survey, this study may not be generalized to reflect all Vietnamese care attendants working in Taiwan. However, the results can serve as a reference to medical institutions operating in a similar capacity. 


\section{Conclusion}

Vietnamese care attendants that have higher levels of occupational stress have lower QOL. Among all factors, "workload and work procedures," "number of daily work hours," and "relationships with management supervisors" were observed to have the greatest influence on QOL. This study suggests that having adequate coping resources, especially understanding the needs of the Vietnamese care attendants in the workplace, may be an important factor for improving their QOL.

\section{References}

[1] Chen MH, Lee SD, Chang HJ, Hsieh PC. Factors associated with nurse aides who willingness to retention: example of long-term care facilities in Taipei. New Taipei Journal of Nursing 2006;8(1):69-77.

[2] Cherry B, Marshall-Gray P, Laurence A, Green A, Valadez A, Scott-Tilley D, Merritt P. Geriatric training academy: Innovative education for certified nurse aides and charge nurses. Joumal of Gerontolgical Nursing2007;33(3):37-44.

[3] Chen NW, Sung HY, Chang SP, Yeh MC, Chang TH. Hospitalized patients satisfaction of care received from foreign care aides. Cheng Ching Medical Journal 2009;5(2): 22-30.

[4] Hsu YC, Wang JJ. Pre-occupational nursing education for Vietnamese foreign laborers. The Journal of Long Term Care 2004;8(1):79-88.

[5] Lin MH, Huang TS, Hsu HC, Wang BY, Lee CY. Exploring sources of working stress, quality of working life, and job satisfaction among aboriginal nurse aides. Chang Gung Nursing 2009;20(2): 180-191.

[6] Ho PL. A study on work stress, coping and physical and mental health status for foreign nurse aides in nursing homes(Unpublished master's thesis).National Taipei College of Nursing, Taiwan, ROC 2005.

[7] Eriksen W, Tambs K, Knardahl S. Work factors and psychological distress in nurses' aides: A prospective cohort study. BMC Public Health 2006;6(290): 1-11.

[8] Huang WY, Hsieh YP. A study on work stress and it's related factors of the foreign nursing aides in nursing-home facilities in Taichung and Changhua area.Journal of Mei Ho Institute of Technology 2010; 29(1): 181-198.

[9] Lin SM. The correlation between job stressor and job satisfaction for nurse aides in long-term care facilities in Shihlin, Peitou areas(Unpublished master's thesis). National Yang-Ming University, Taipei City, Taiwan, ROC 2000 .

[10] Lin YW, Chang YW, Tsai CC. Job strain and health-related quality of life of hospital employees: Case of a medical center in Tai-Chung. Taiwan Journal of Public Health 2004; 23(2): 108-120.

[11] Lin CC, Wang SC, Kan TC, Temg JW. Leisure behavior, quality of working life, job stress and job performance of the employees of national universities.Journal of Sport, Leisure and Hospitality Research 2008;3(3): 1-26.
[12] Liu CH, Yen HW, Chiou SY, Liao LL, Liao JY, Hung HJ. Relation between working status and health-related quality of life among health educators in junior high schools. Taiwan Journal of Public Health 2007;26(3): 229-240.

[13] Sehlen S, Vordermark D, Schäfer C, Herschbach P, Bayerl A, Pigorsch S, Geinitz H. Job stress and job satisfaction of physicians, radiographers, nurses and physicists working in radiotherapy: A multicenter analysis by the DEGRO Quality of Life Work Group.Radiation Oncology 2009;4(6): 4-6.

[14] Lin CC, Tseng MY. A survey of job satisfaction and its related factors among nurse aides. The Journal of Long Term Care 2005; 9(4): 349-360.

[15] Liu LF. Job satisfaction among care assistants in nursing homes: An exploratory study conducted in three cities in Southern Taiwan. Journal of Leader University 2005;2(2):87-100.

[16] Yang X, Ge C, Hu B, Chi T, Wang L. Relationship between quality of life and occupational stress among teachers. Public Health 2009;123(11):750-755.

[17] Yu YJ, Hung SW, Wu YK, Tsai LC, Wang HM, Lin CJ. Job satisfaction and quality of life among hospital nurses in the Yunlin-Chiayi area. The Journal of Nursing 2008;55(2): 29-38.

[18] Schaefer JA, Moos RH. Relationship, task and system stressors in the health care workplace. Journal of Community \& Applied Social Psychology 1993;3(4): 285298.

[19] Lu JF, Tseng HM, Tsai YJ. Assessment of health-related quality of life in Taiwan (I): Development and psychometric testing of SF-36 Taiwan version. Taiwan Journal of Public Health 2003;22(6): 501-511.

[20] Feng CK, Li CY, Chou CM, Huang LL. A cross-sectional survey of stress and social support among caregivers working in long-term care institutions. Fu-Jen Journal of Medicine 2003; 1(1):35-46.

[21] Sakai Y, AkiyamaT, Miyake Y, Kawamura Y, Tsuda H, Kurabayashi L, Tominaga M, Noda T, Akiskal K. Temperament and job stress in Japanese company employees. Journal of AffectiveDisorders 2005; 85:101-112.

[22] Christine K, Christin B. Determinants of subjective quality of life in depressed patients: The role of self-esteem, response styles, and social support.Journal of Affective Disorders 2005;86(2-3): 205-213.

[23] Lin YW, Peng JY, Lin ZW, Hong JY.. Clarifying the relationship between healthy organizations and employees' quality of work life: The case of a regional teaching hospital in Taiwan. The Journal of Health Science 2006;8(1): 20-35.

[24] Eriksen W, Bruusgaard D, Knardahl S. Work factors as predictors of sickness absence: A three month prospective study of nurses'aides. Occupational and Environmental Medicine 2003;60(4): 271-278.

[25] Li SL, Chuang YH, Chen HY, Lin SY. A narrative of newly employed nurses facing work stress in a Kaohsiung teaching hospital. Journal of Nursing and Healthcare Research 2009; 5(2): 83-90.

[26] Liang WM, Kuo HW. Effects of workplace conditions on Taiwanese workers' quality of life. Mid-Taiwan Journal of 
Medicine 2002;7(4): 206-214.

[27] TohLC, LinLP, LinJD. A study on the job stress and the health status of the staff in long term care facilities. Taiwan Journal Of Gerontological Health Research 2007;3(2):73-82. 